\title{
Hybrid Metamaterial for the Secondary Radar Antenna System
}

\author{
Peerasan Khamsalee* Piyaporn Mesawad · Rangsan Wongsan
}

\begin{abstract}
This paper proposes the gain enhancement of dual-band and dual-polarized asymmetric horn antenna for the secondary radar system using hybrid metamaterial techniques. The hybrid metamaterial is comprised of the structures of woodpile electromagnetic bandgap (EBG) for gain enhancement of the primary main beam of the radar system at the operating frequency of $1,300 \mathrm{MHz}$ with horizontal polarization; and the wire medium structure that is placed beside the EBG structure for gain improvement of the identification friend or foe (IFF) main beam, which is operated at the center frequency of $1,060 \mathrm{MHz}$ with vertical polarization. Meanwhile, the cooperated structures have to function to control the directions of the primary and IFF main beams retaining at $0^{\circ}$ and $6^{\circ}$, respectively, too. When the hybrid metamaterial structure is placed at the front of an asymmetric horn's aperture, with suitable parameters and optimized spacing, it is found to increase the gains of the two beams compared to the single asymmetric horn around $3 \mathrm{~dB}$ and retain the directions of original main beams. The comparison of the results between simulation and measurement, such as the reflected power $\left(S_{11}\right)$, gain, and radiation patterns, are in good agreement.
\end{abstract}

Key Words: Gain Enlargement, Electromagnetic Band Gap, Horn Antenna, Secondary Radar, Wire Medium.

\section{INTRODUCTION}

Radio detection and ranging (RADAR) is a method of using electromagnetic waves to remotely sense the velocity, position, and identifying characteristics of targets [1]. The secondary radar system is another type of radar that is widely used for detecting distant targets. The secondary radar system has two main operating systems. The first deals with detecting and reporting the position of a target by transmitting a periodic pulse signal to the target and receiving the reflected signal back. The second is used to identify the detected target using the identification friend or foe (IFF) system, in which the IFF signal is transmitted to the target and the answered message is received back. To avoid interference between two adjacent frequency bands of the radar system, orthogonal polarization has been used in the rectangular horn antenna as a feeder of the reflector antenna that is commonly used in radar systems.

There are two ways to improve the radiated efficiency of the radar system: (1) increase the transmitting power or (2) enhance the gain of the antenna. In this paper, we focus on the gain improvement of the primary feeder of the reflector antenna as an alternative to enlarging the dimension of the reflector. The primary feeder of DR-172 ADV medium-altitude radar, which is our reference radar, uses one asymmetric rectangular horn for feeding electromagnetic waves to the reflector with the dualfrequency bands $(1,215-1,365 \mathrm{MHz}$ for target detection and $1,030-1,090 \mathrm{MHz}$ for target identification), dual-polarization, and different main beam direction.

This is an Open-Access article distributed under the terms of the Creative Commons Attribution Non-Commercial License (http://creativecommons.org/licenses/by-nc/4.0) which permits unrestricted non-commercial use, distribution, and reproduction in any medium, provided the original work is properly cited.

(c) Copyright The Korean Institute of Electromagnetic Engineering and Science. All Rights Reserved. 
In the current research, the methods for performance enhancement of the antenna were reported by using the new technology, which is called a metamaterial structure since it can increase the radiation efficiency of microwave antennas $[2,3]$. The properties of the metamaterial result from the composition and the combination of materials, where the structural dimension is considerably smaller compared to the given wavelength. Furthermore, the permeability and permittivity of metamaterial are zero or near-zero, and the reflective index is a negative quantity $[4,5]$. The electromagnetic bandgap (EBG) is a type of the metamaterials with a periodic structure; these materials are known as photonic crystals [6] or photonic bandgap materials. However, the EBG structures are usually realized by a periodic arrangement of dielectric material and metallic conductors. In general, the EBG can be classified into three groups according to a geometric configuration: one-dimensional transmission line, two-dimensional planar surface, and three-dimensional volumetric structure. The woodpile EBG is the selected technique for increasing the gain of an asymmetric rectangular horn in this paper since its 3D structure is very suitable for locating at the front of the horn's aperture to receive the EM energy from the aperture and pass through its own structure into space. The woodpile EBG structures were used as the resonator antennas by locating them above the ground plane of the different feeding antennas (i.e., the double slot and microstrip patch antennas). This paper reports that the woodpile EBG material was able to create highly directional patterns [7]. In addition, the woodpile EBG was designed as a cylindrical structure to cover the monopole antenna, resulting in an increase in the gain of the monopole antenna [8].

Furthermore, from $[9,10]$, the quadratic-shaped woodpile EBG structure was used to enhance the gain of any horn antenna by its placement at the front of the horn's aperture with optimized spacing. In 2016, Kamphikul and Wongsan [11] continued to develop the cavity of curved woodpile EBG structure for the enhancement of the gain of the slot array antenna instead of increasing the number of slots on the array. Nevertheless, the wire medium is another metamaterial that has a woodpile EBG-type structure $[12,13]$. The electromagnetic properties of the wire medium structure designed at the plasma frequency are near zero permittivity or permeability [14]. Tomaz et al. [15] proposed the use of the wire medium structure for decreasing the sidelobe level of the radiation patterns and increasing the directivity of the traditional horn antenna by using five layers of wire embedded in the Styrofoam dielectric. The wire medium was also applied to the horn antenna to reduce the length of the horn, whereas the aperture size of the horn did not change [16]. Later studies $[17,18]$ reported on the influence of different wire medium structures placed at the front of the aperture of the horn antenna, resulting in higher gain and lower sidelobe level in traditional horns. From the literature review, we found that both metamaterial structures installed in front of the aperture can increase the gain of the horn antenna. Therefore, this paper proposes the gain enhancement of dual-band and dual-polarized asymmetric horn antenna for secondary radar system using a hybrid metamaterial technique. The proposed hybrid structure comprises woodpile EBG to encourage the first beam for the target detection of the radar system at operating frequency $1,300 \mathrm{MHz}$ with horizontal polarization and a wire medium for improving the second beam for the IFF system at operating frequency $1,060 \mathrm{MHz}$ with vertical polarization. Simultaneously, the proposed structure is capable of treating both main beam directions as the same as the original asymmetric horn provided.

First, the design and configurations of a horn antenna and the hybrid metamaterial are described in Sections II. Second, the simulated results and discussion of all parts of the structure are demonstrated in Section III. Third, the measured results of the established prototype antenna are shown in Section IV. Fourth, the simulated results of the horn antenna and the hybrid metamaterial with the reflector are shown in Section V. Lastly, the conclusions are given in Section VI.

\section{HORN ANTENNA AND HYBRID METAMATERIAL}

\section{CONFIGURATIONS}

\section{An Asymmetric Rectangular Horn Antenna}

Fig. 1 shows the structure of the asymmetric horn antenna, which was modelled from an original horn of DR-172 ADV radar system by Daimler-Chrysler Aerospace, Germany, and was designed to support the dual-frequency band and dualpolarization to function as a feeder of the main reflector antenna of this radar system. The first beam of the feeding horn was designed for target detection of the radar system at an operating frequency of 1,300 MHz with horizontal polarization. Simultaneously, the second beam of this horn was designed for transceiving the IFF signals between the radar station and targets (aircraft) with vertical polarization at frequencies of $1,030 \mathrm{MHz}$ (Tx) and 1,090 MHz (Rx). Moreover, this feeding horn was also designed to control two main beams for target detection and IFF transceiving of the radar systems with different directions at $0^{\circ}$ and $6^{\circ}$, respectively. It also provides the gain for target detection beam at $10.85 \mathrm{dBi}$, and the gains for the IFF beam at 12.39 $\mathrm{dBi}(\mathrm{Tx})$ and $12.51 \mathrm{dBi}(\mathrm{Rx})$, respectively.

\section{Hybrid Metamaterial Configurations}

To improve the gains of this asymmetric horn antenna, we use the combination of woodpile EBG and wire medium structures, which is the hybrid metamaterial that controls the performance of the electromagnetic wave. However, to design the 


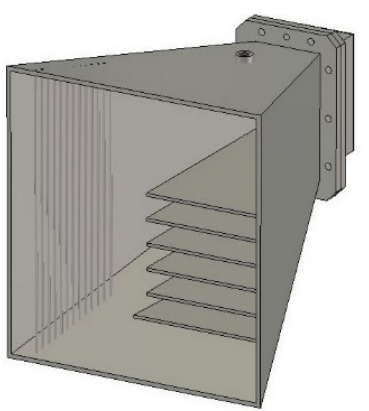

(a)

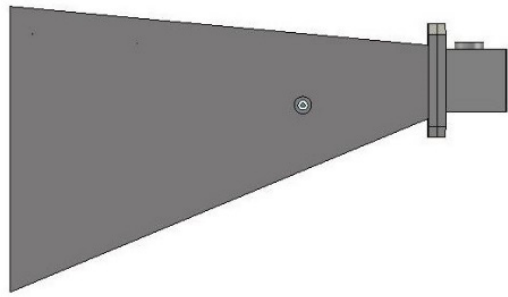

(b)

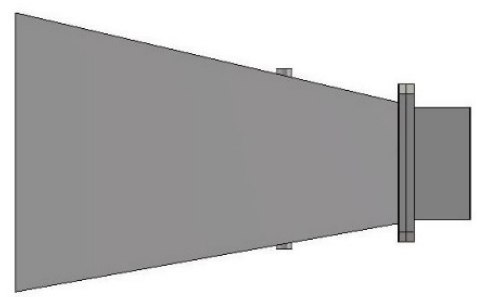

(c)

Fig. 1. Structure of asymmetric horn antenna: (a) perspective view, (b) top view, and (c) side view.

proposed hybrid metamaterial, we separated the structure into two parts, namely, structures of woodpile EBG and wire medium, respectively. First, the woodpile EBG structure is designed to be the 3D periodic structure made from the dielectric material (superlene rods with $\varepsilon_{r}=3.3$ ), as shown in Fig. 2, for the enhancement of the directive gain of the target detection beam at the operating frequency of $1,300 \mathrm{MHz}$. This EBG structure is placed at the front of an asymmetric horn with the suitable position and spacing to fully support the illumination of waves from the boresight of the target detection beam from the horn aperture. In Fig. 2, the given parameters of the unit cell of the woodpile EBG structure are the height of rods $\left(h_{1}\right)$ in layer 1 and 3 , the height of rods $\left(b_{2}\right)$ in layer 2 and 4 , the length of rods $(a)$, the width of rods $(w)$ and the total height of unit cell $(b)$. These proper parameters of the unit cell are optimized and simulated using CST Studio Suite Software. In the first step, we simulate the impact of the length of rods $(a)$ in a unit cell versus the reflected power $\left(S_{11}\right)$ to consider the resonant frequency response, as shown in Fig. 3. It is found that the resonant frequency increases as the rod length increase. Fig. 4 shows the comparison of the simulated reflected power versus the different heights of two rods $\left(b_{1}\right)$ in layers 1 and 3 . We found that the resonant frequency increases as the rod height decreases. In addition, if the height of the other two rods $\left(b_{2}\right)$ in layers 2 and 4 is decreased, the resonant frequency is shifted to a higher frequency,

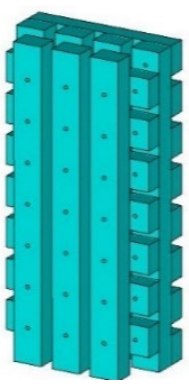

(a)

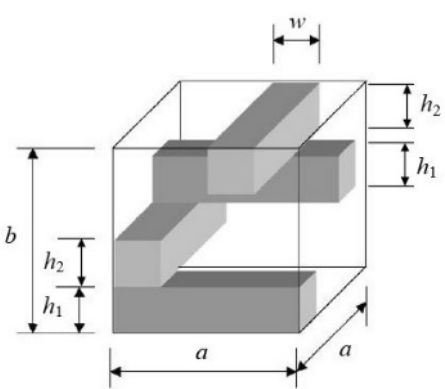

(b)
Fig. 2. Structure of woodpile EBG: (a) periodic structure and (b) unit cell.

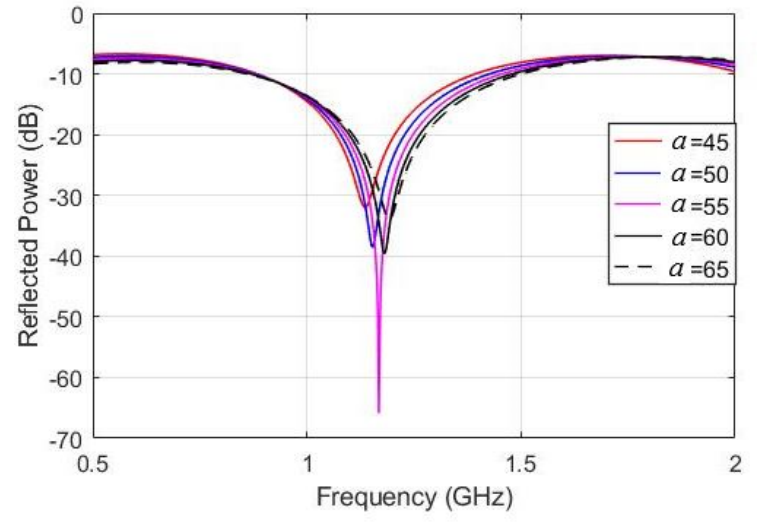

Fig. 3. The simulated reflected power $\left(S_{11}\right)$ versus the different lengths $(a)$ of dielectric rods.

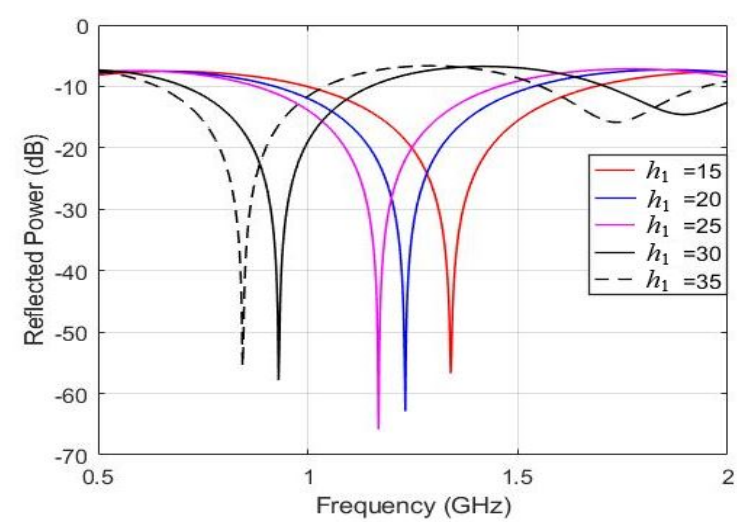

Fig. 4. The simulated reflected power $\left(S_{11}\right)$ versus the different heights $\left(h_{1}\right)$ of dielectric rods in layers 1 and 3.

as shown in Fig. 5. Next, the influence of the width on the resonant frequency is considered, as shown in Fig. 6. We found that the resonant frequency decrease as the rod width decreases. Lastly, the most appropriate parameters of a unit cell of the woodpile EBG structure are summarized as follows: $a=55 \mathrm{~mm}$, $h_{1}=25 \mathrm{~mm}, h_{2}=30 \mathrm{~mm}, b=110 \mathrm{~mm}$, and $w=40 \mathrm{~mm}$.

From a single unit cell in Fig. 2(b), the 21 unit cells are assembled by arranging the dielectric rods in a $3 \times 7$ pattern, as shown in Fig. 2(a). With a $450 \mathrm{~mm}$ spacing between the EBG 


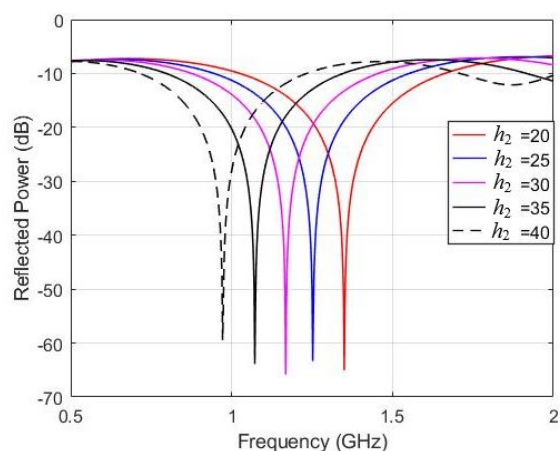

Fig. 5. The simulated reflected power $\left(S_{11}\right)$ versus the different heights $\left(h_{2}\right)$ of dielectric rods in layers 2 and 4 .

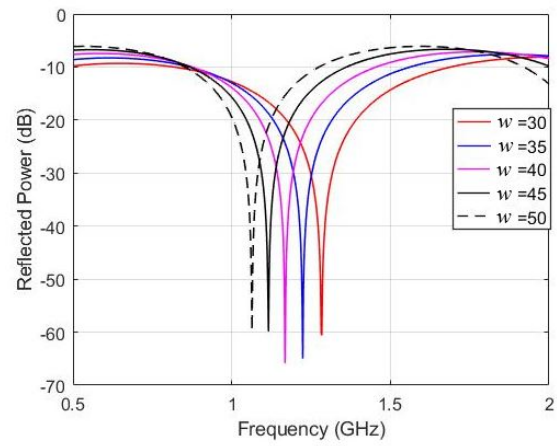

Fig. 6. The simulated reflected power $\left(S_{11}\right)$ versus the different widths $(w)$ of dielectric rods.

structure and horn aperture, we found that the structure yields a bandgap of $1.05 \mathrm{GHz}$ to $1.37 \mathrm{GHz}$ at $S_{11}=-15 \mathrm{~dB}$, covering the resonant frequency of $1,060 / 1,300 \mathrm{MHz}$ and the overall bandwidth of the horn antenna (Fig. 7). However, we found that the directions of the two beams did not conform to the requirements.

Therefore, to compensate for the diffraction of waves on the left side of the EBG structure, we extended and varied the length of the dielectric rods $\left(W_{\mathrm{E}}\right)$ in layers 1 and 3 , as displayed in Fig. 8, to control the different directions of the two beams, as illustrated in Table 1 . We found that with the length of dielec-

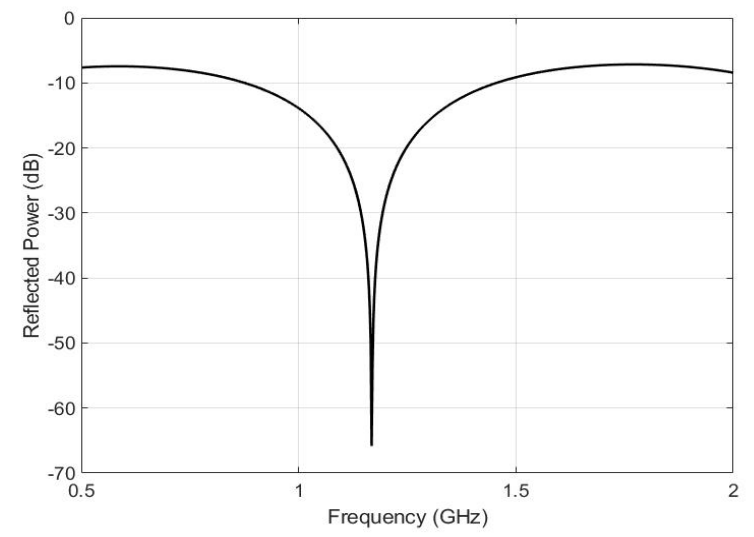

Fig. 7. The simulated reflected power $\left(S_{11}\right)$ of woodpile EBG structure before extending the length of the dielectric rods.

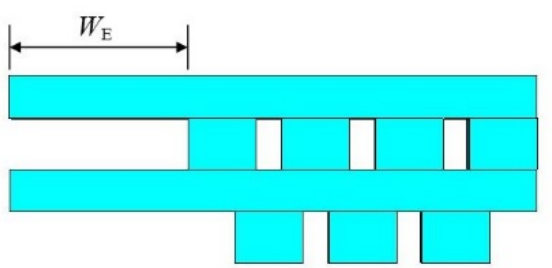

Fig. 8. Structure of woodpile EBG with $3 \times 7$ dielectric rods (top view).

Table 1. The 1st simulated results of radar beam directions

\begin{tabular}{cccc}
\hline \multirow{2}{*}{$\begin{array}{c}\text { Length } \\
(\mathrm{mm})\end{array}$} & \multicolumn{3}{c}{ Directions of radar beams (o) } \\
\cline { 2 - 4 } & $1,030 \mathrm{MHz}$ & $1,090 \mathrm{MHz}$ & $1,300 \mathrm{MHz}$ \\
\hline 50 & 6 (left) & 6 (left) & 6 (left) \\
100 & 6 (left) & 6 (left) & 3 (left) \\
105 & 6 (left) & 6 (left) & 0 \\
110 & 4 (left) & 5 (left) & 1 (right) \\
115 & 4 (left) & 5 (left) & 2 (right) \\
120 & 4 (left) & 5 (left) & 3 (right) \\
\hline
\end{tabular}

tric rods $\left(W_{\mathrm{E}}\right)$ at $105 \mathrm{~mm}$, the direction of the target detection and IFF beams of radar are controlled at $0^{\circ}$ and $6^{\circ}$, respectively.

To support the IFF beam operated at $1,030 \mathrm{MHz}$ and 1,090 $\mathrm{MHz}$ frequencies with vertical polarization, the wire medium structure uses two layers of the dielectric rods installed at the right side of the EBG structure to improve the gain of the IFF signal (Fig. 9). The parameters of the wire medium structure are as follows: the rod width $\left(W_{\mathrm{w}}\right)$, the rod thickness $\left(H_{\mathrm{W}}\right)$, the rod height $\left(W_{\mathrm{L}}\right)$, the spacing of adjacent rods $\left(W_{\mathrm{S}}\right)$ in the same layer, and the spacing between two layers $\left(H_{\mathrm{S}}\right)$, which are optimized by CST simulation. From the simulation results, the appropriate parameters are following: $W_{\mathrm{w}}=45 \mathrm{~mm}, H_{\mathrm{W}}=30 \mathrm{~mm}, W_{\mathrm{L}}=$ $535 \mathrm{~mm}, W_{\mathrm{S}}=16 \mathrm{~mm}$, and $H_{\mathrm{S}}=25 \mathrm{~mm}$.

In Fig. 10, the EBG and wire medium structures with the optimized dimension are combined and simulated to observe the beam behavior.

Table 2 illustrates the directions of radar beams for the IFF signal and target detection when located the combined structure at the front of the horn aperture. We found that the effect from an assembly of two structures causes the directions of both beams to change since the diffraction of waves that have different polarizations at the junction of arrangements are not truly isolated, as displayed in Fig. 11. Consequently, the beam direc-

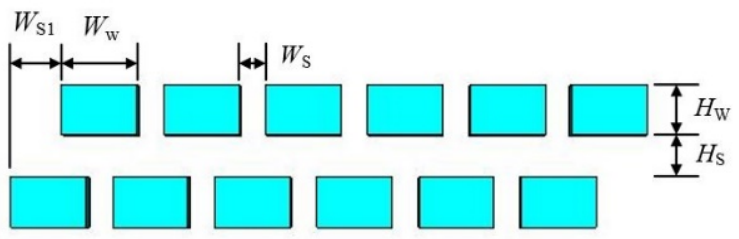

Fig. 9. Structure of wire medium (top view). 


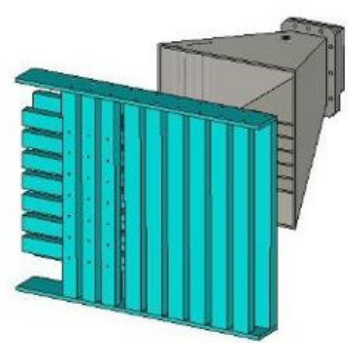

Fig. 10. Combination of EBG and wire medium structures.

Table 2. The 2nd simulated results of radar beam directions

\begin{tabular}{cccc}
\hline \multirow{2}{*}{$\begin{array}{c}\text { Length } \\
(\mathrm{mm})\end{array}$} & \multicolumn{3}{c}{ Directions of radar beams (o) } \\
\cline { 2 - 4 } & $1,030 \mathrm{MHz}$ & $1,090 \mathrm{MHz}$ & $1,300 \mathrm{MHz}$ \\
\hline 60 & 6 (left) & 6 (left) & 2 (right) \\
65 & 6 (left) & 6 (left) & 2 (right) \\
70 & 6 (left) & 6 (left) & 0 \\
75 & 6 (left) & 6 (left) & 1 (left) \\
80 & 6 (left) & 6 (left) & 3 (left) \\
\hline
\end{tabular}

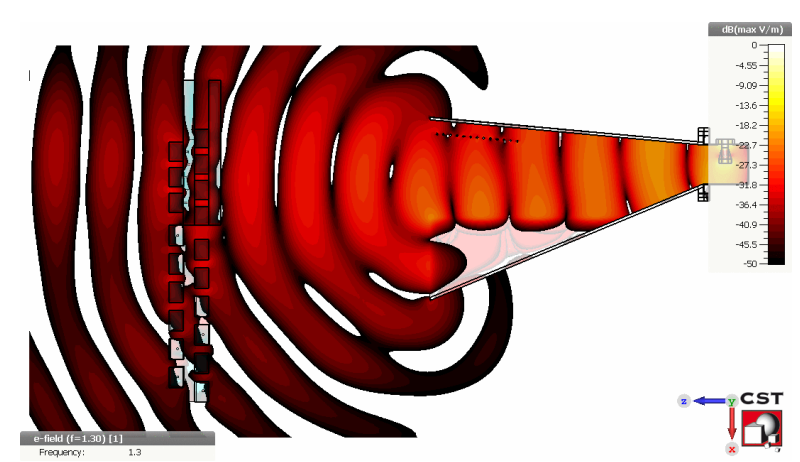

Fig. 11. Show the diffraction of the electric field (at 1,300 MHz) pass through some part of the wire medium structure.

tion of $1,300 \mathrm{MHz}$ is shifted right from $0^{\circ}$ to $3^{\circ}$. To reduce the diffraction of the electromagnetic waves from the target detection beam to the wire medium structure, we designed an array of $1 \times 6$ metallic rods with a $9.53 \mathrm{~mm}$ diameter lying in the horizontal line according to a polarization of $1,300 \mathrm{MHz}$ beam with rod spacing $\left(S_{\mathrm{S}}\right)$. From the simulation, it is found that the proper spacing between the center of adjacent rods is $35.6 \mathrm{~mm}$ by placing the one ends of rods at the front of the horn aperture with suitable distance around $4 \mathrm{~mm}$, as shown in Fig. 12. Furthermore, the length of metallic rods $\left(L_{\mathrm{S}}\right)$ is varied to meet the requirements for the directions of two different beams (Table 2). It is observed that the most suitable length $\left(L_{\mathrm{S}}\right)$ is $70 \mathrm{~mm}$, providing the beam direction of the frequency of $1,300 \mathrm{MHz}$ back to $0^{\circ}$. However, this rod array could not float in the air without the holder; therefore, we designed the metallic rod holder using the superlene sheet by forming a suitable shape and not obstructing the travelling waves from horn aperture, as shown in Fig. 12(b).

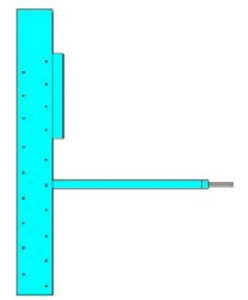

(a)

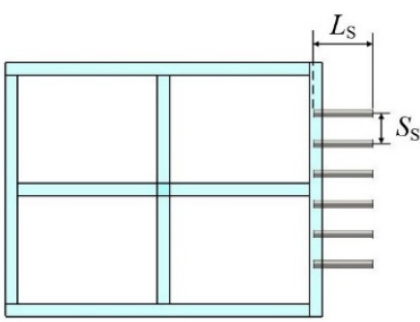

(b)
Fig. 12. The $1 \times 6$ metallic rods with a dielectric holder: (a) topview and $(\mathrm{b})$ side view.

\section{SIMULATED RESULTS AND DISCUSSIONS}

The configuration of an asymmetric horn antenna combined with a hybrid metamaterial structure is shown in Fig. 13. The essential parameters of the original asymmetric horn antenna and asymmetric horn antenna with the proposed hybrid metamaterial, such as the reflected power for $1,300 \mathrm{MHz}\left(S_{11}\right)$ and $1,060 \mathrm{MHz}\left(S_{22}\right)$, the radiation patterns and gain are simulated using CST software.

Fig. 14 shows the comparison for the reflected power of the target detection beam $\left(S_{11}\right)$ and the IFF beam $\left(S_{22}\right)$ at the center frequencies of $1,300 \mathrm{MHz}$ and $1,060 \mathrm{MHz}$, respectively. The frequency responses of the asymmetric horn are still similar after adding the hybrid metamaterial structure with a bandwidth of $1,084-1,504 \mathrm{MHz}$ and $1,026-1,098 \mathrm{MHz}$ at $S_{11}=-15 \mathrm{~dB}$, as shown in Fig. 14(a) and (b), respectively.

The simulated results of the normalized radiation pattern at the operating frequencies of $1,030 \mathrm{MHz}, 1,090 \mathrm{MHz}$, and

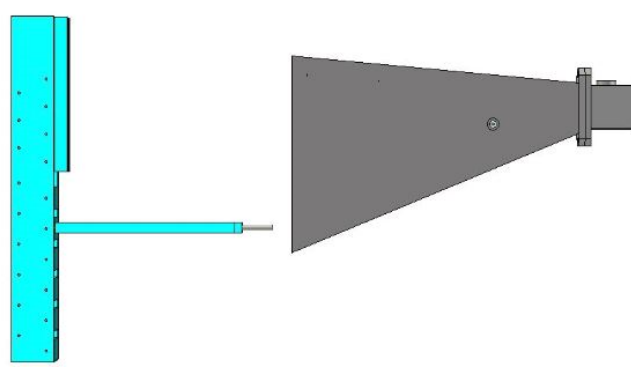

(a)

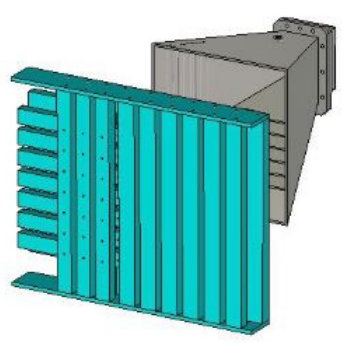

(b)

Fig. 13. The configuration of the proposed antenna: (a) top view and (b) perspective view. 


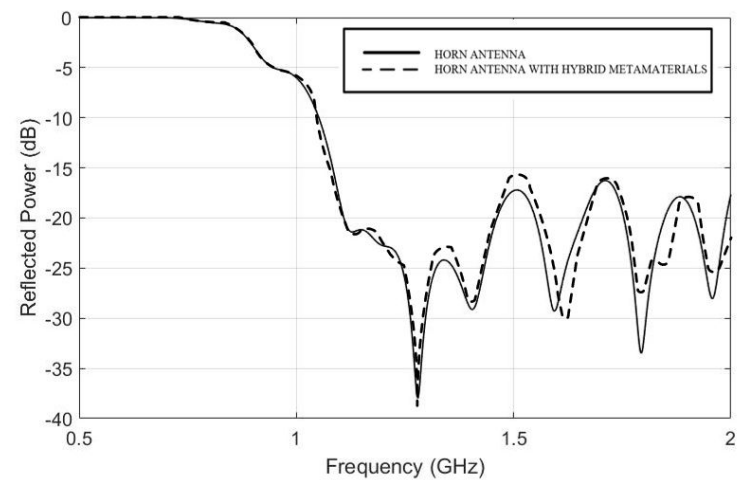

(a)

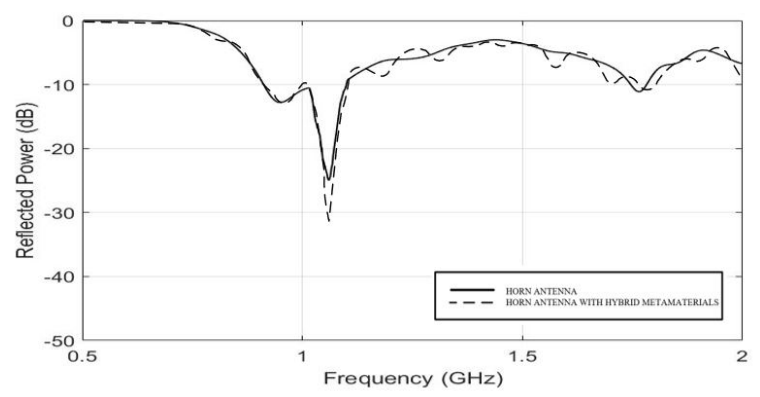

(b)

Fig. 14. The comparison of reflected power: (a) for target detection beam $\left(S_{11}\right)$ and (b) for IFF beam $\left(S_{22}\right)$.

Table 3. Simulation parameters of the single asymmetric horn without/with the hybrid metamaterial

\begin{tabular}{|c|c|c|c|c|c|}
\hline & \multirow[t]{2}{*}{ Parameter } & \multicolumn{2}{|c|}{ Horn antenna } & \multicolumn{2}{|c|}{$\begin{array}{c}\text { Horn antenna } \\
\text { with hybrid } \\
\text { MM }\end{array}$} \\
\hline & & $\mathrm{E}$ & $\mathrm{H}$ & $\mathrm{E}$ & $\mathrm{H}$ \\
\hline 1,030 & Gain (dBi) & \multicolumn{2}{|c|}{12.39} & \multicolumn{2}{|c|}{15.64} \\
\hline \multirow[t]{2}{*}{$\mathrm{MHz}$} & Main beam dir. (o) & 1 & 6 & 0 & 6 \\
\hline & HPBW (o) & 36.5 & 49.2 & 25.2 & 28.2 \\
\hline 1,090 & Gain (dBi) & \multicolumn{2}{|c|}{12.51} & \multicolumn{2}{|c|}{15.55} \\
\hline \multirow[t]{2}{*}{$\mathrm{MHz}$} & Main beam dir. (o) & 1 & 6 & 0 & 6 \\
\hline & HPBW (o) & 35.9 & 48.1 & 24.1 & 26.8 \\
\hline 1,300 & Gain (dBi) & \multicolumn{2}{|c|}{10.85} & \multicolumn{2}{|c|}{14.04} \\
\hline \multirow[t]{2}{*}{$\mathrm{MHz}$} & Main beam dir. (o) & 0 & 0 & 0 & 1 \\
\hline & HPBW (o) & 62.0 & 43.9 & 32.0 & 26.4 \\
\hline
\end{tabular}

$\mathrm{MM}=$ metamaterial, dir. $=$ direction, $\mathrm{E}=\mathrm{E}$-plane, $\mathrm{H}=\mathrm{H}$-plane.

1,300 $\mathrm{MHz}$ in both $\mathrm{E}$ - and $\mathrm{H}$-plane are compared and shown in Fig. 15. We found that the hybrid metamaterial can control the difference of beam directions of both systems, which was retained at $0^{\circ}$ in the E-plane of the target detection beam and $6^{\circ}$ in the $\mathrm{H}$-plane of the IFF beam. However, the half-power beamwidths (HPBWs) obtained from the proposed hybrid metamaterial are somewhat narrower than those of the single asymmetric horn. As a result, the gains achieved from the hybrid metamaterial at the operating frequencies of $1,030 \mathrm{MHz}, 1,090$
$\mathrm{MHz}$, and 1,300 MHz are $15.64 \mathrm{dBi}, 15.55 \mathrm{dBi}$, and $14.04 \mathrm{dBi}$, respectively. It is shown that the hybrid metamaterial can increase the gains around $3 \mathrm{~dB}$ at all operating frequencies, as shown in Table 3. The simulated results of the radiation patterns of both cases are displayed in Fig. 15.

\section{MEASURED RESULTS AND DISCUSSIONS}

The prototype of an asymmetric horn antenna with a hybrid metamaterial was assembled and tested in the semi-anechoic chamber at Electrical and Electronic Products Testing Center (PTEC), Thailand, as shown in Fig. 16. The simulated and measured reflected powers $\left(S_{11}, S_{22}\right)$ for the prototype are shown in Fig. 17. The measured and simulated results are in good agreement. The bandwidth of the given operating frequencies is retained according to the requirement. In Fig. 18, the normalized radiation patterns obtained from the simulation and measurement are compared. We found that the measured patterns are somewhat similar to the simulated results. It is also observed that the measured results of the beam directions are retained in the desired direction, as per the simulated results. In Table 4, we mention the obtained parameters from simulation and measurement for comparison. The measured gains obtained from an asymmetric horn with the proposed hybrid metamaterial are higher than that of a single horn around $3.05 \mathrm{dBi}, 3.03 \mathrm{dBi}$, and $3.25 \mathrm{dBi}$. In contrast, the simulated gains are higher than approximately $3.25 \mathrm{dBi}, 3.04 \mathrm{dBi}$, and $3.19 \mathrm{dBi}$ at the frequencies of $1,030 \mathrm{MHz}, 1,090 \mathrm{MHz}$, and 1,300 $\mathrm{MHz}$, respectively. Moreover, we also compared the simulated and measured results of the sidelobe level and HPBW in both the E- and H-planes (Table 4).

In Fig. 18, the simulated and measured radiation patterns in $\mathrm{E}$ - and $\mathrm{H}$-planes at the operating frequencies of $1,030 \mathrm{MHz}$, $1,090 \mathrm{MHz}$, and 1,300 MHz are compared and displayed. The shapes of the radiation patterns in each plane of each frequency are somewhat similar. Nevertheless, we found that the measured radiation patterns in some planes yielding the sidelobe level were slightly higher than that of the simulated results such as $\mathrm{H}$-plane of 1,030 MHz, E-plane of 1,090 MHz, and H-plane of $1,300 \mathrm{MHz}$, as shown in Fig. 18(b), (c), and (f), respectively.

\section{HORn ANTENNA AND HyBRID METAMATERIAL WITH REFLECTOR}

To verify that the modified feeding antenna is assembled from an asymmetric horn and the proposed hybrid metamaterial using the CST simulation software. We modelled an offset-fed reflector by importing the structure of $8.3 \mathrm{~m}$ in diameter, and $\mathrm{f} / \mathrm{D}$ ratio equals to 0.34 of the paraboloidal reflector with a horn feed at the position of its focal point (around $2.8 \mathrm{~m}$ from the 


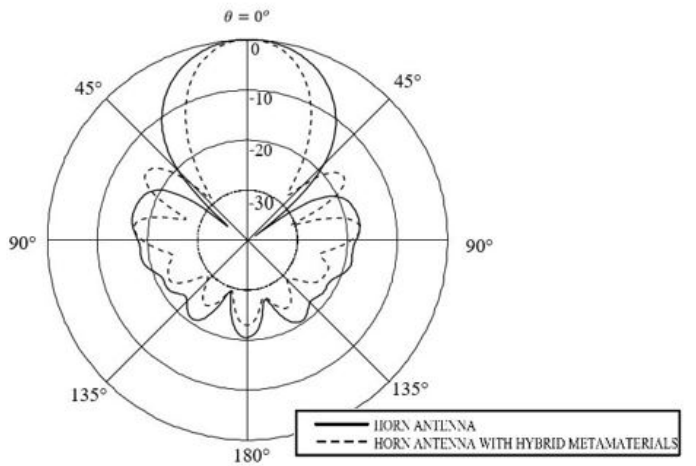

(a)

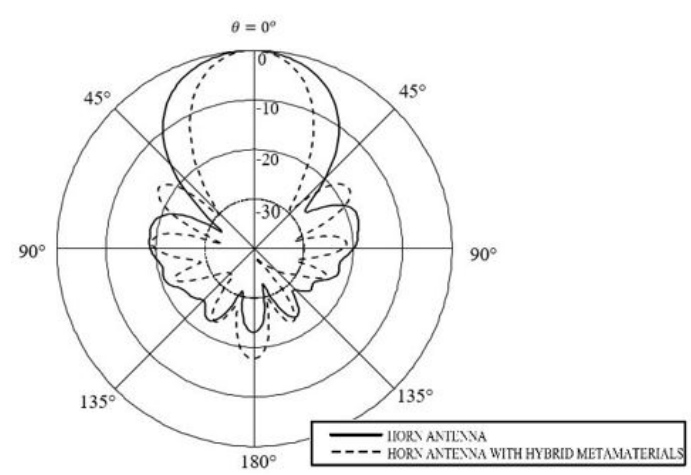

(c)

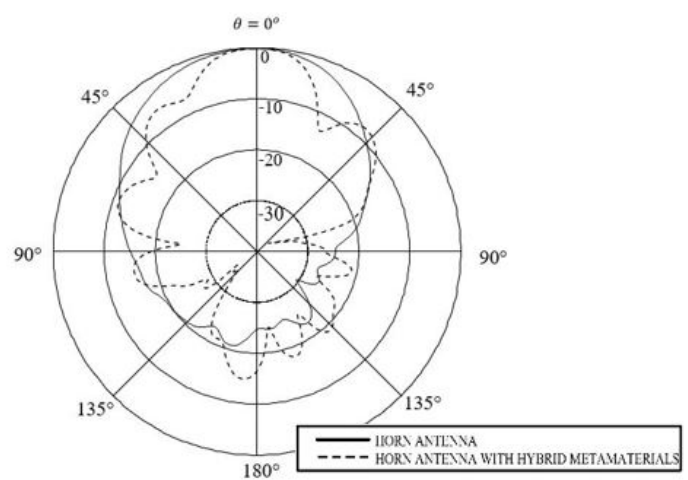

(e)

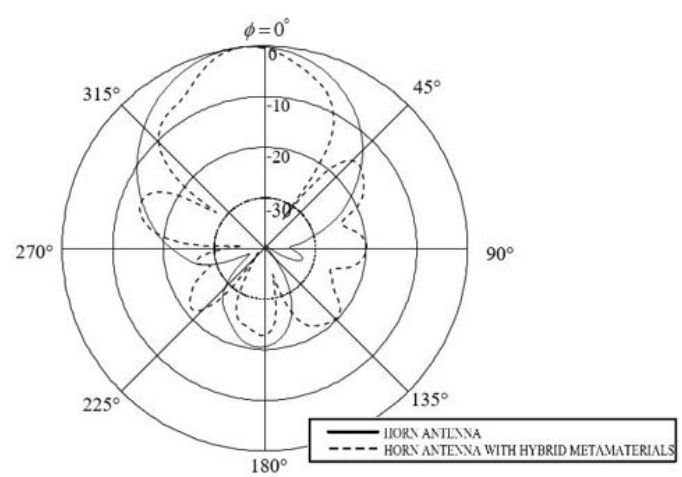

(b)

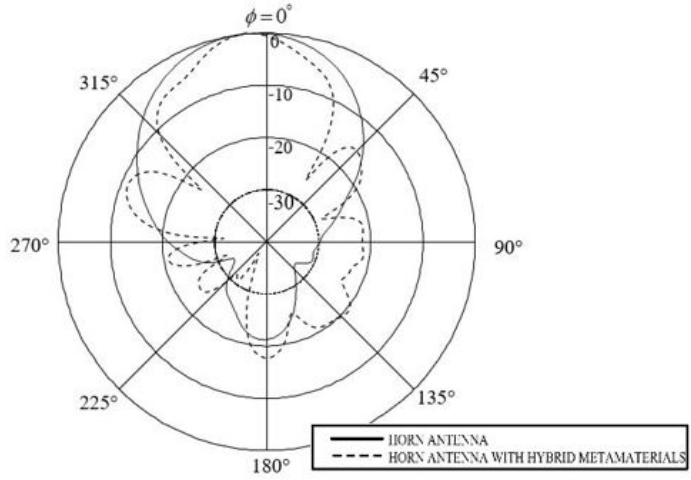

(d)

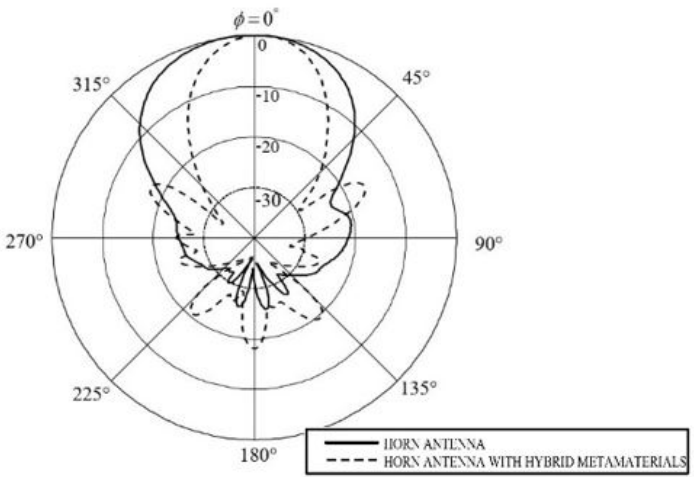

(f)

Fig. 15. Comparison of simulated radiation patterns of a single asymmetric horn without/with hybrid metamaterial: (a, c, e) E-planes and (b, d, f) H-planes at operating frequency of $1,030 \mathrm{MHz}, 1,090 \mathrm{MHz}$, and 1,300 MHz, respectively.

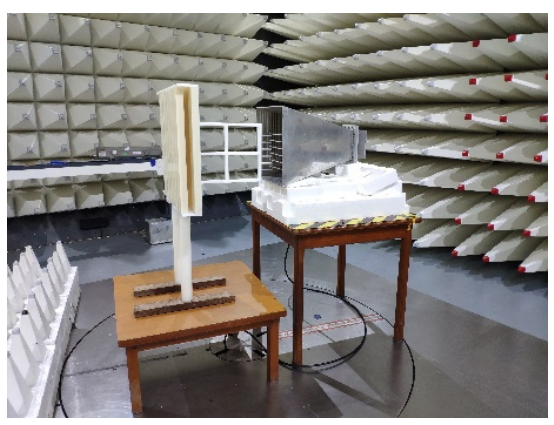

(a)

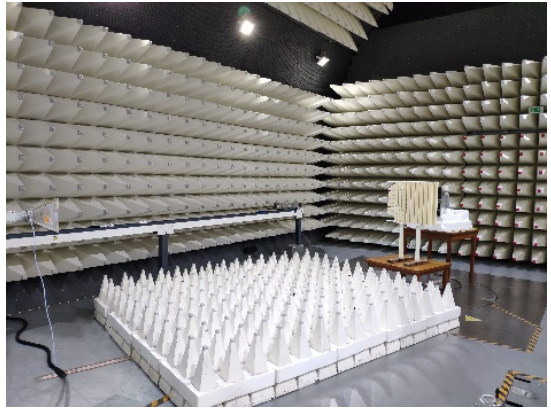

(b)

Fig. 16. The measurement setup for fabricated hybrid metamaterial with an asymmetric horn antenna in anechoic chamber: (a) side view and (b) perspective view. 


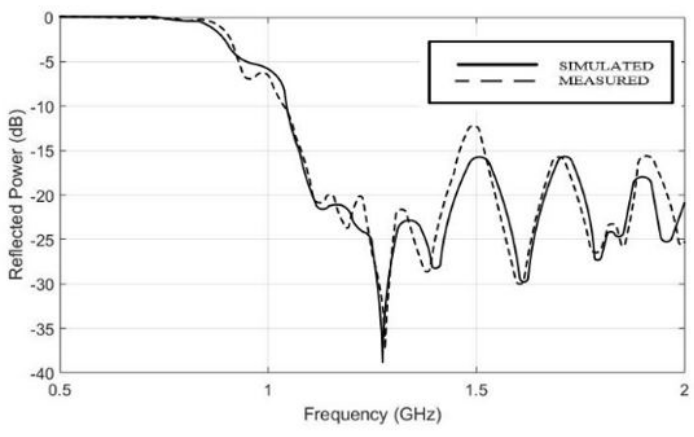

(a)

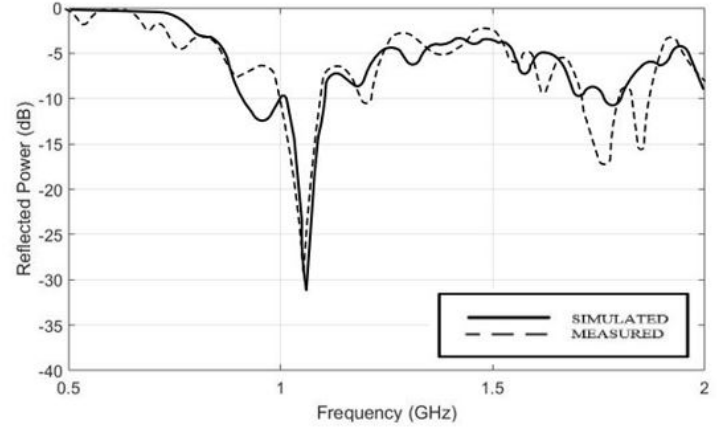

(b)

Fig. 17. The comparison of reflected power: (a) main $\operatorname{radar}\left(S_{11}\right)$ and (b) IFF systems $\left(S_{22}\right)$.

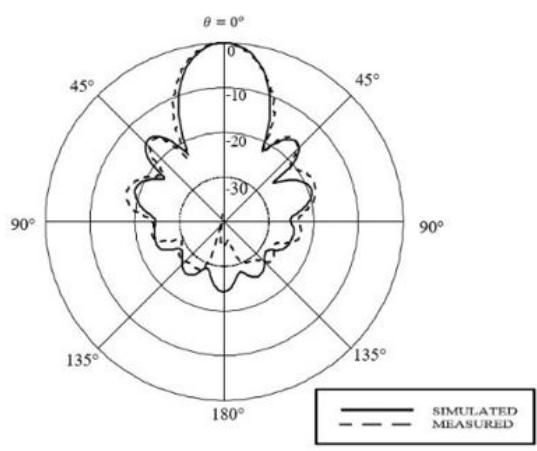

(a)

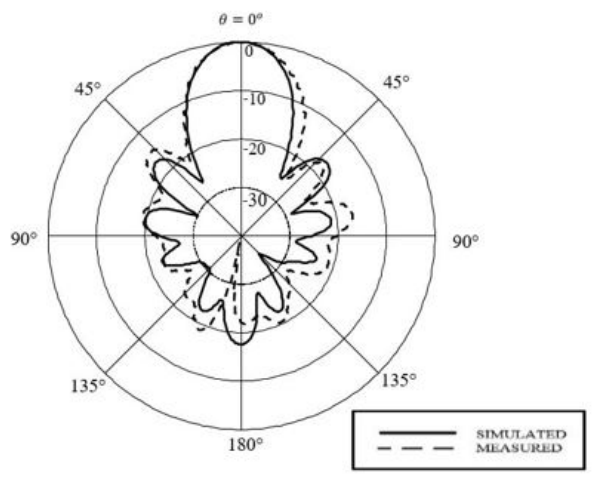

(c)

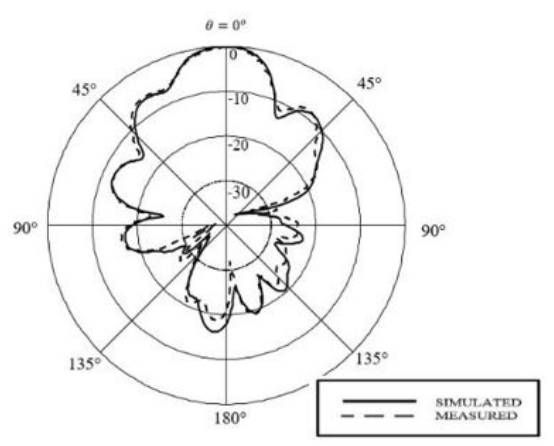

(e)

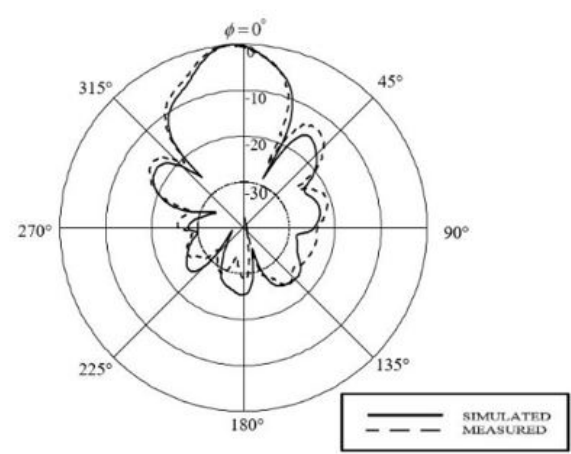

(b)

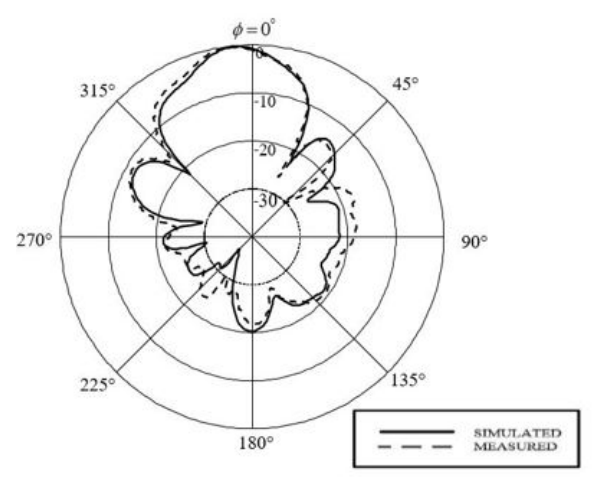

(d)

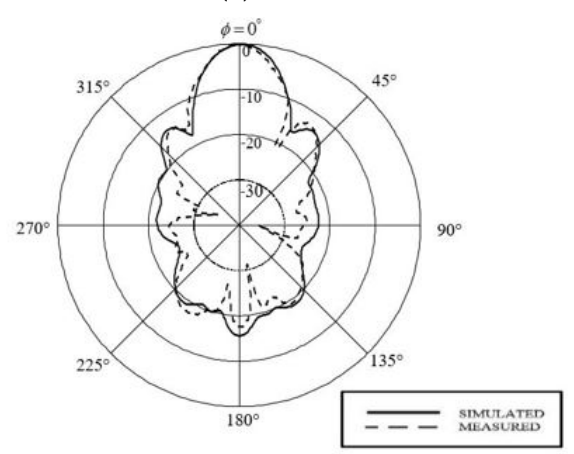

(f)

Fig. 18. Comparison of simulated and measured radiation patterns of an asymmetric horn with hybrid metamaterial: (a, c, e) E-planes and

(b, d, f) H-planes at operating frequency of $1,030 \mathrm{MHz}, 1,090 \mathrm{MHz}$, and 1,300 MHz, respectively.

vertex) from the CST library. Only the upper portion of the reflector is selected and modified by referring to the real struc- ture of an offset-fed reflector of the DR-172 ADV radar system having the aperture dimensions of approximately $7.2 \mathrm{~m}$ and 3.5 
Table 4. Comparison of simulated and measured parameters of single asymmetric horn without/with hybrid metamaterial

\begin{tabular}{|c|c|c|c|c|c|c|c|c|c|}
\hline & \multirow{3}{*}{ Parameter } & \multicolumn{4}{|c|}{ Horn antenna } & \multicolumn{4}{|c|}{ Horn antenna with hybrid MM } \\
\hline & & \multicolumn{2}{|c|}{ Simulation } & \multicolumn{2}{|c|}{ Measurement } & \multicolumn{2}{|c|}{ Simulation } & \multicolumn{2}{|c|}{ Measurement } \\
\hline & & $\mathrm{E}$ & $\mathrm{H}$ & $\mathrm{E}$ & $\mathrm{H}$ & $\mathrm{E}$ & $\mathrm{H}$ & $\mathrm{E}$ & $\mathrm{H}$ \\
\hline \multirow[t]{4}{*}{$1,030 \mathrm{MHz}$} & Gain $(\mathrm{dBi})$ & \multicolumn{2}{|c|}{12.39} & \multicolumn{2}{|c|}{11.16} & \multicolumn{2}{|c|}{15.65} & \multicolumn{2}{|c|}{14.21} \\
\hline & Main beam dir. (o) & 1 & 6 & 0 & 6 & 0 & 6 & 0 & 6 \\
\hline & HPBW (o) & 36.5 & 49.2 & 40.0 & 51.0 & 25.2 & 28.2 & 25.0 & 29.0 \\
\hline & SSLs $(\mathrm{dB})$ & -18.4 & - & -16.63 & -24.48 & -17.0 & -15.5 & -17.97 & -13.32 \\
\hline \multirow[t]{4}{*}{$1,090 \mathrm{MHz}$} & Gain $(\mathrm{dBi})$ & \multicolumn{2}{|c|}{12.51} & \multicolumn{2}{|c|}{11.12} & \multicolumn{2}{|c|}{15.55} & \multicolumn{2}{|c|}{14.15} \\
\hline & Main beam dir. (o) & 1 & 6 & 2 & 6 & 0 & 6 & 0 & 6 \\
\hline & HPBW (o) & 35.9 & 48.1 & 39.0 & 52.0 & 24.1 & 26.8 & 25.0 & 29.0 \\
\hline & SSLs $(\mathrm{dB})$ & -18.2 & - & -15.46 & - & -16.8 & -12.4 & -14.95 & -13.32 \\
\hline \multirow[t]{4}{*}{$1,300 \mathrm{MHz}$} & Gain $(\mathrm{dBi})$ & \multicolumn{2}{|c|}{10.85} & \multicolumn{2}{|c|}{10.50} & \multicolumn{2}{|c|}{14.04} & \multicolumn{2}{|c|}{13.75} \\
\hline & Main beam dir. (o) & 0 & 0 & 0 & 1 & 0 & 1 & 0 & 0 \\
\hline & HPBW (o) & 62.0 & 43.9 & 65.0 & 40.0 & 32.0 & 26.4 & 29.0 & 23.0 \\
\hline & SSLs (dB) & - & -17.6 & - & -29.79 & -6.8 & -13.9 & -8.57 & -13.71 \\
\hline
\end{tabular}

$\mathrm{MM}=$ metamaterial, dir. $=$ direction, $\mathrm{E}=\mathrm{E}$-plane, $\mathrm{H}=\mathrm{H}$-plane, $\mathrm{SSL}=$ sidelobe level.

$\mathrm{m}$ in width and height, respectively. The radiation pattern of an asymmetric horn with hybrid metamaterial is offset, as displayed in Fig. 19. Therefore, it illuminates only the upper portion of the reflector. Even though the metamaterial structure is installed at the front of the asymmetric and its top edge is over the top edge of the horn at approximately $0.49 \mathrm{~m}$, the proposed feeder still is in a position that is well clear of the main beam. Therefore, no obstruction occurs [19]. However, in the process of simulating the performance of the offset-fed reflector antenna excited by an asymmetric horn with hybrid metamaterial, we can use one feature of CST simulation software. That is the far-field sources that are available as excitation for the integral equation solver instead of simulation by using a model of horn directly. Therefore, the far-field source data obtained from the simulated patterns, which are illustrated and explained in Section III, is imported and functions as the new source for the primary reflector, as described with graphic images in Fig. 19(b)-(g).

Even though the HPBWs obtained from the proposed hybrid metamaterial are somewhat narrower and the sidelobes occur more than those of the single asymmetric horn, they are still limited in the envelope of the original main beams obtained from a single horn. To verify that the appearance of the sidelobes does not affect the sidelobes resulting from waves reflection on the surface of the offset-fed reflector. The simulated results, as shown in Fig. 20, can be considered.

In Fig. 20, we found that the elevation beam angles with vertical polarization for $1,060 \mathrm{MHz}$ of the center frequency for the IFF band are lifted at $10^{\circ}$, which is the same as that of the target detection beam at $1,300 \mathrm{MHz}$ with horizontal polarization, as shown in Fig. 20(a) and (d), respectively. The beam directions in the azimuthal angle of the IFF and target detection systems are maintained at $6^{\circ}$ and $0^{\circ}$, which followed the requirement, as shown in Fig. 20(b) and (c), respectively. Furthermore, the obtained sidelobes of all cases are in the standard of the Federal Communications Commission $(\leq-20 \mathrm{~dB})$.

We found that the gains of a single asymmetric horn with the reflector are approximately $23.31 \mathrm{dBi}, 23.56 \mathrm{dBi}$, and $21.74 \mathrm{dBi}$ at the operating frequency of $1,030 \mathrm{MHz}, 1,090 \mathrm{MHz}$, and $1,300 \mathrm{MHz}$, respectively. Once the hybrid metamaterial is added to the front of the horn aperture, we found that the reflector antenna provides higher gains of approximately $26.60 \mathrm{dBi}$, $26.76 \mathrm{dBi}$, and $25.43 \mathrm{dBi}$ at frequencies of $1,030 \mathrm{MHz}, 1,090$ $\mathrm{MHz}$, and 1,300 MHz, respectively. Therefore, we can summarize that the offset-fed reflector antenna for the radar system with the hybrid metamaterial can increase the gain of each frequency by approximately $3 \mathrm{~dB}(3.29 \mathrm{~dB}, 3.2 \mathrm{~dB}$, and $3.69 \mathrm{~dB}$ at $1,030 \mathrm{MHz}, 1,090 \mathrm{MHz}$, and 1,300 MHz, respectively).

\section{CONCLUSION}

We proposed the gain enhancement of the dual-band and dual-polarized asymmetric horn antenna for the secondary radar system using original horn from the DR-172 ADV radar system as a reference. The hybrid metamaterial structure was designed for placing at the front of the horn aperture to increase the gains of this asymmetric horn. The proposed architecture consists of two types of metamaterials structure: the woodpile EBG structure and wire medium work together with the origin- 


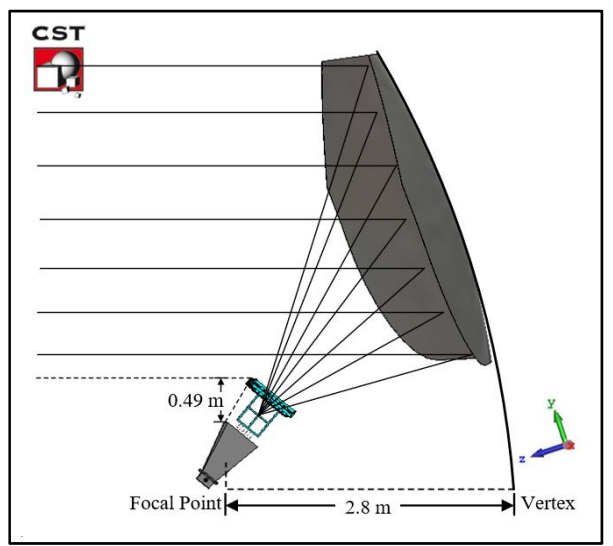

(a)

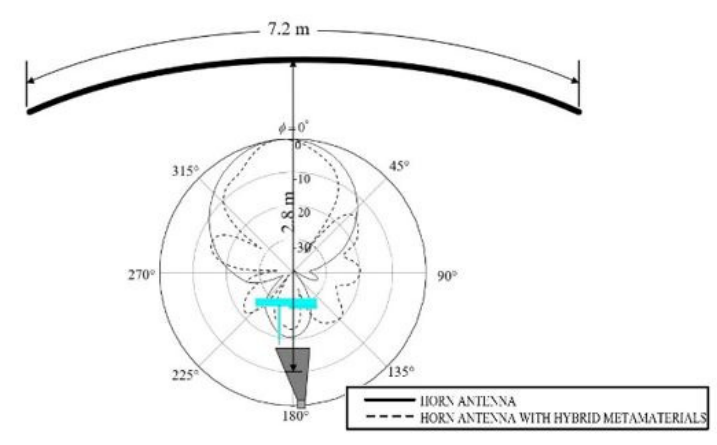

(c)

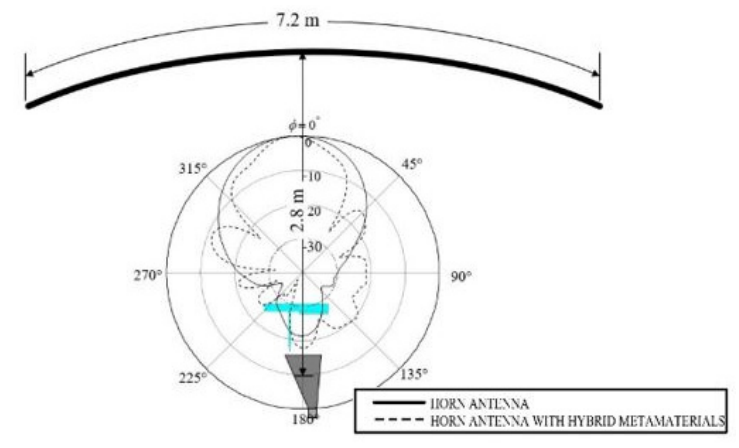

(e)

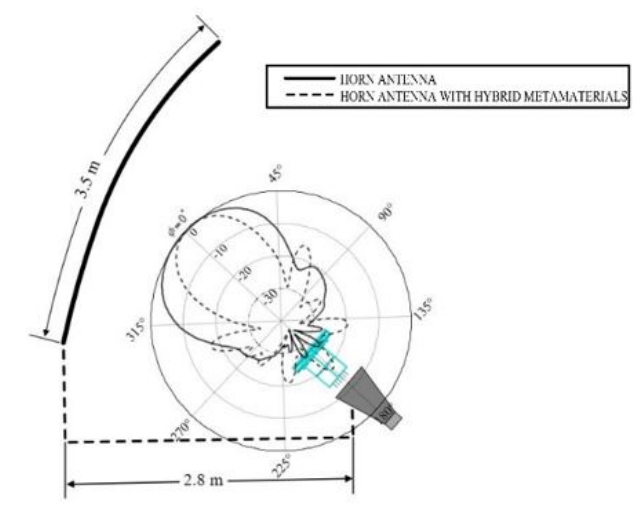

(g)

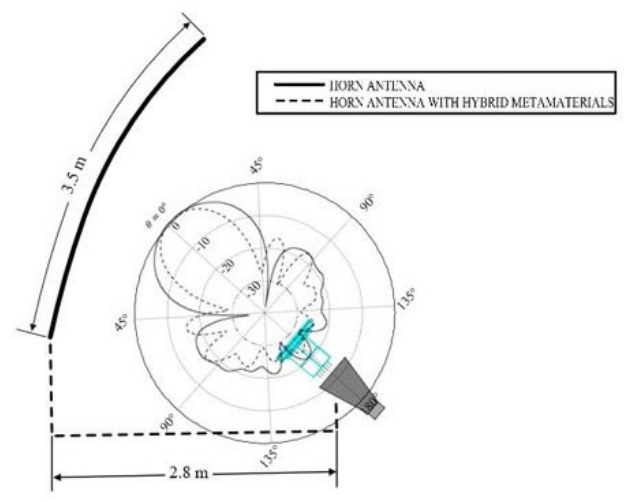

(b)

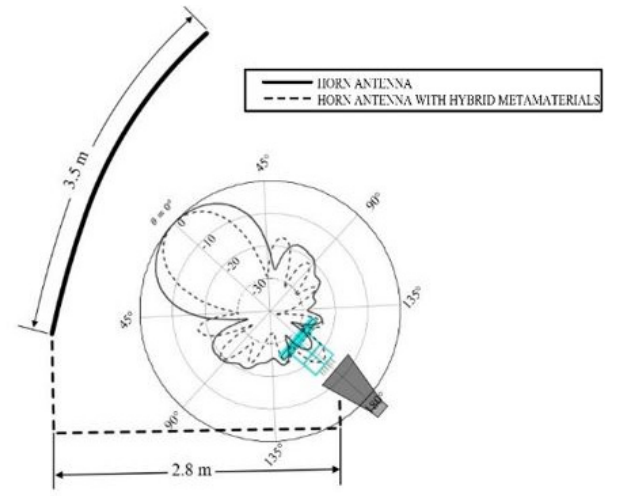

(d)

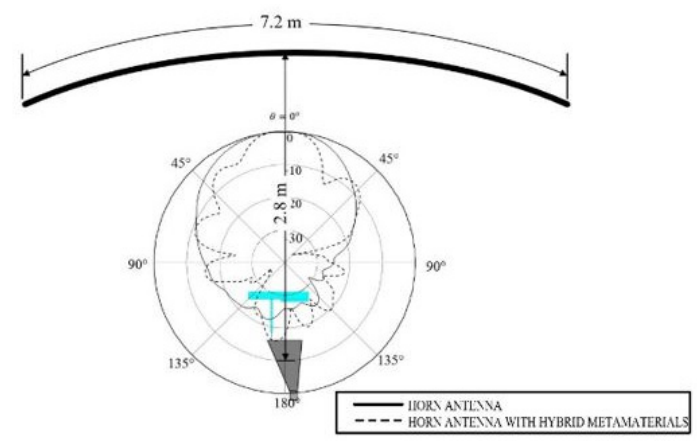

(f)

Fig. 19. Model and graphic images of the primary reflector with offset-fed asymmetric horn and hybrid metamaterial by exciting with the simulated far-field sources: (a) model of offset-fed reflector feed by the proposed feeder and (b-g) show excitation sources for the primary reflector in both planes at frequencies $1,030 \mathrm{MHz}, 1,090 \mathrm{MHz}$, and 1,300 MHz. (a) Perspective view and (b, d, g) side views, and (c, e, f) top views. 


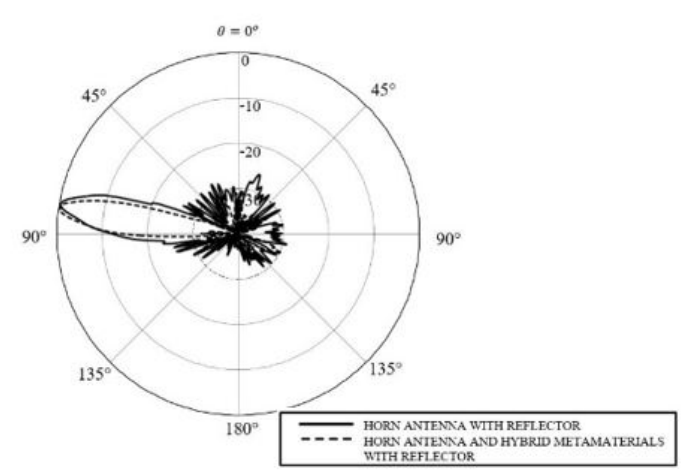

(a)

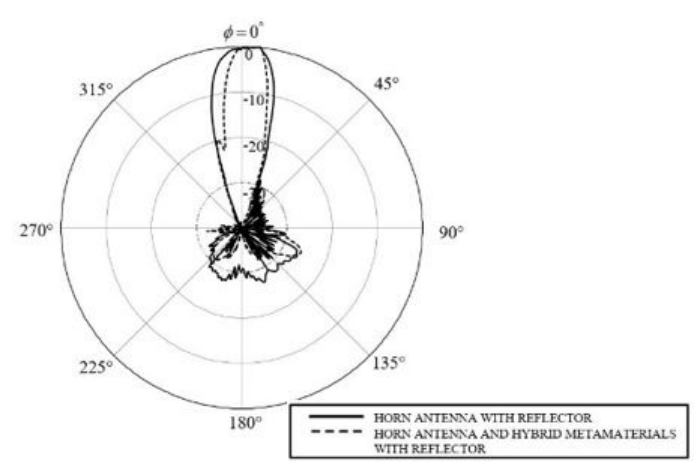

(b)

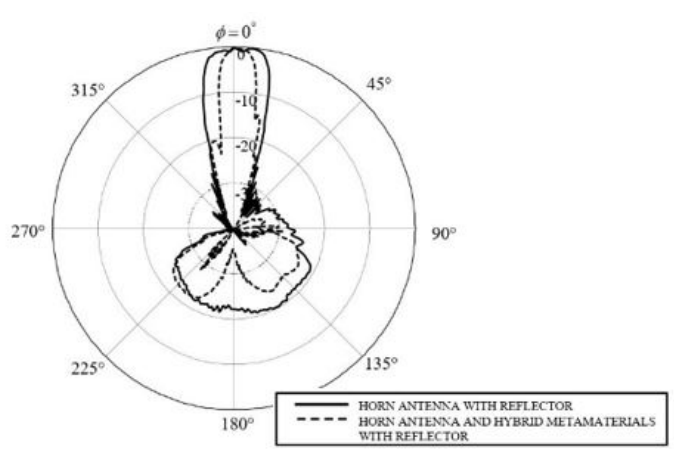

(c)

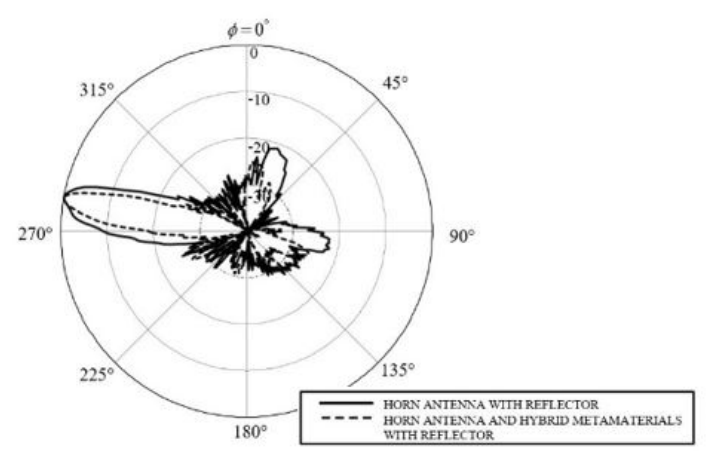

(d)

Fig. 20. Comparison of simulated radiation patterns of an offsetfed reflector antenna excited by an asymmetric horn without/with the hybrid metamaterial structure. (a) Elevation angle for E-plane of $1,060 \mathrm{MHz}$, (b) azimuthal angle for $\mathrm{H}$-plane of 1,060 MHz, (c) azimuthal angle for E-plane of $1,300 \mathrm{MHz}$, and (d) elevation angle for $\mathrm{H}$-plane of 1,300 MHz. nal asymmetric horn. The gain enhancement of the target detection beam at the operating frequency of $1,300 \mathrm{MHz}$ with horizontal polarization was achieved by using the property of the woodpile EBG structure. The wire medium structure improved the gain of the IFF beam at frequencies of $1,030 \mathrm{MHz}$ $(\mathrm{Tx})$ and $1,090 \mathrm{MHz}(\mathrm{Rx})$, respectively, with vertical polarization. The simulated and measured results for the asymmetric horn without/with the proposed hybrid metamaterial structure, such as the gains, radiation patterns, and reflected power, were in good agreement. Importantly, the proposed hybrid metamaterial structure can improve the gains of both beams by slightly more than $3 \mathrm{~dB}$ and retain the direction of both beams with reference to the original beam directions obtained from the reference horn modelled.

This work was supported by the Research Department institute of Engineering, Suranaree University of Technology, Nakhonratchasima, Thailand.

\section{REFERENCES}

[1] B. Edde, Radar: Principles, Technology, Applications. Englewood Cliffs, NJ: Prentice Hall, 1993.

[2] Y. J. Lee, J. Yeo, R. Mittra, and W. S. Park, "Application of electromagnetic bandgap (EBG) superstrates with controllable defects for a class of patch antennas as spatial angular filters," IEEE Transactions on Antennas and Propagation, vol. 53, no. 1, pp. 224-235, 2005.

[3] F. Frezza, L. Pajewski, E. Piuzzi, C. Ponti, and G. Schettini, "Analysis and experimental characterization of an alumina woodpile-covered planar antenna," in Proceedings of the 40th European Microwave Conference, Paris, France, 2010, pp. 200-203.

[4] D. R. Smith, W. J. Padilla, D. C. Vier, S. C. Nemat-Nasser, and S. Schultz, "Composite medium with simultaneously negative permeability and permittivity," Physical Review Letters, vol. 84, no. 18, pp. 4184-4187, 2000.

[5] N. Fang and X. Zhang, "Imaging properties of a metamaterial superlens," in Proceedings of the 2nd IEEE Conference on Nanotechnology, Washington, DC, 2002, pp. 225-228.

[6] J. D. Joannopoulos, S. G. Johnson, J. N. Winn, and R. D. Meade, Photonic Crystals: Molding the Flow of Light. Princeton, NJ: Princeton University Press, 2008.

[7] A. R. Weily, L. Horvath, K. P. Esselle, B. C. Sanders, and T. S. Bird, "A planar resonator antenna based on a woodpile EBG material," IEEE Transactions on Antennas and Propagation, vol. 53, no. 1, pp. 216-223, 2005.

[8] Y. Lee, X. Lu, Y. Hao, S. Yang, J. R. Evans, and C. G. Parini, "Low-profile directive millimeter-wave antennas using free- 
formed three-dimensional (3-D) electromagnetic bandgap structures," IEEE Transactions on Antennas and Propagation, vol. 57, no. 10, pp. 2893-2903, 2009.

[9] S. Kampeephat, P. Krachodnok, and R. Wongsan, "Gain improvement for conventional rectangular horn antenna with additional EBG structure," in Proceedings of 2014 11th International Conference on Electrical Engineering/Electronics, Computer, Telecommunications and Information Technology (ECTI-CON), Nakhon Ratchasima, Thailand, 2014, pp. 1-4.

[10] R. Wongsan, P. Krachodnok, S. Kampeephat, P. Kamphikul, "Gain enhancement for conventional circular horn antenna by using EBG technique," in Proceedings of 2015 12th International Conference on Electrical Engineering/Electronics, Computer, Telecommunications and Information Technology (ECTI-CON), Hua Hin, Thailand, 2015, pp. 1-4.

[11] P. Kamphikul and R. Wongsan, "Gain improvement for rectangular horn antenna by using curved-woodpile metamaterial," in Proceedings of 2016 13th International Conference on Electrical Engineering/Electronics, Computer, Telecommunications and Information Technology (ECTI-CON), Chiang Mai, Thailand, 2016, pp. 1-4.

[12] P. Burghignoli, G. Lovat, F. Capolino, D. R. Jackson, and D. R. Wilton, "Directive leaky-wave radiation from a dipole source in a wire-medium slab," IEEE Transactions on Antennas and Propagation, vol. 56, no. 5, pp. 1329-1339, 2008.

[13] P. Burghignoli, G. Lovat, F. Capolino, D. R. Jackson, and D. R. Wilton, "Modal propagation and excitation on a wiremedium slab," IEEE Transactions on Microwave Theory and

Peerasan Khamsalee

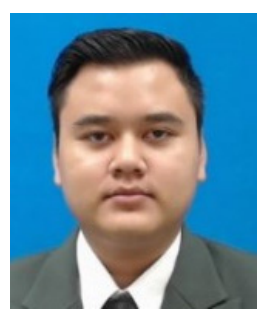

was born in Nakhonratchasima, Thailand, 1991. He earned his Bachelor of Engineering degree in Telecommunication Engineering from Suranaree University of Technology, Thailand, in 2013, as well as his Master of Engineering degree in Telecommunication Engineering at Suranaree University of Technology in 2015. He is currently working toward his doctorate in telecommunication engineering at $\mathrm{Su}^{-}$ ranaree University of Technology. His research interests include electromagnetic theory, antenna engineering, and metamaterial technology.
Techniques, vol. 56, no. 5, pp. 1112-1124, 2008.

[14] E. Forati and G. W. Hanson, "Scattering from isotropic connected wire medium metamaterials: three-, two-, and one-dimensional cases," IEEE Transactions on Antennas and Propagation, vol. 61, no. 7, pp. 3564-3574, 2013.

[15] A. Tomaz, J. J. Barroso, P. J. Castro, and A. J. F. Orlando, "Experimental investigation on the radiation pattern of a horn antenna loaded by a wire medium," in Proceedings of 2013 IEEE Antennas and Propagation Society International Symposium (APSURSI), Orlando, FL, 2013, pp. 958-959.

[16] M. K. T. Al-Nuaimi, W. Hong, and Y. Zhang, "Design of high-directivity compact-size conical horn lens antenna," IEEE Antennas and Wireless Propagation Letters, vol. 13, pp. 467-470, 2014.

[17] P. Duangtang, P. Mesawad, and R. Wongsan, "Gain improvement of conical horn antennas by adding wire medium structure," in Proceedings of 2016 13th International Conference on Electrical Engineering/Electronics, Computer, Telecommunications and Information Technology (ECTICON), Chiang Mai, Thailand, 2016, pp. 1-5.

[18] S. Kampeephat, P. Kamphikul, and R. Wongsan, "Gain improvement for conventional rectangular horn antenna with additional two-layer wire medium structure," in Proceedings of 2017 Progress in Electromagnetics Research Symposium-Fall (PIERS-FALL), Singapore, 2017, pp. 2493-2497.

[19] D. J. Brain and A. W. Rudge, "Efficient satellite antennas," Electronics and Power, vol. 30, no. 1, pp. 51-56, 1984.

Piyaporn Mesawad

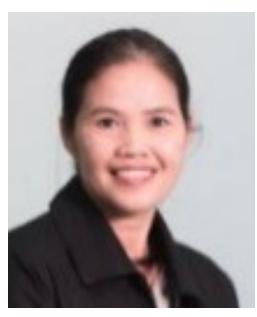

received her Bachelor of Engineering degree in telecommunication engineering at Suranaree University of Technology, Thailand, in 1997, as well as her Master of Engineering degree in electrical engineering from Chulalongkorn University, Thailand, in 2001. She then received her Doctor of Engineering degree in telecommunication engineering at $\mathrm{Su}^{-}$ ranaree University of Technology, Thailand, in 2008. She is a reviewer for the IEEE Conference. Her research interests are electromagnetic theory, antenna engineering, and the electromagnetic band gap. Associate Professor Dr. Piyaporn is a member of the Electrical Engineering/Electronic, Computer, Telecommunication, and Information Technology Association (ECTI) and the Institute of Electronics, Information, and Communication Engineers (IEICE). 


\section{Rangsan Wongsan}

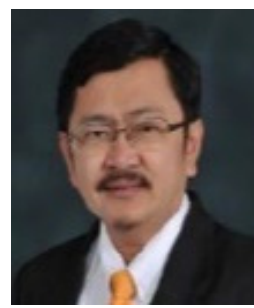

received his Bachelor of Engineering degree in electronics engineering at Rajamangala Institute of Technology (RIT) in 1989, as well as his Master of Engineering degree in electrical engineering from King Mongkut's Institute of Technology North Bangkok (KMITNB) in 1994. He then received his Doctor of Engineering degree in electrical engineering at King Mongkut's Institute of Technology Ladkrabang (KMITL) in 2003. He is currently a reviewer of many journals related to electromagnetic applications. His research focuses on antenna theory and electromagnetic applications. Currently, his research interests are the utilization of metamaterials for efficient improvement of conventional antennas and microwave devices. Associate Professor Dr. Rangsan is a member of the Electrical Engineering/Electronic, Computer, Telecommunication, and Information Technology Association (ECTI) and the Institute of Electronics, Information, and Communication Engineers (IEICE). 